\title{
Muscle tuning and preferred movement path: do we need a paradigm shift or should we redefine the old? - comment on Nigg et al.
}

\author{
Benedicte Vanwanseele ${ }^{1, *}$, Xianyi Zhang ${ }^{1}$ \& Kurt Schütte ${ }^{1}$ \\ 1 Department of Movement Sciences, KU Leuven, Leuven, Belgium \\ * Corresponding author: Department of Movement Science, University of Leuven, Tervuursevest 101, 3001 Leuven, Belgium, \\ Tel: +32 (0) 16-32 9123 \\ Email: benedicte.vanwanseele@kuleuven.be
}

\section{COMMENTARY}

\section{Article History:}

Submitted $8^{\text {th }}$ March 2018

Accepted $9^{\text {th }}$ March 2018

Published $18^{\text {th }}$ April 2018

Handling Editor:

Markus Tilp

Karl-Franzens-University Graz, Austria

Editor-in-Chief:

Martin Kopp

University of Innsbruck, Austria

\section{ABSTRACT}

In the feature paper "Muscle tuning and preferred movement path - a paradigm shift", Benno Nigg and colleagues discuss that the impact and pronation paradigm should be abandoned as there is not enough biomechanical and epidemiological evidence supporting these paradigms. We agree that the paradigms, as defined in the paper, are currently not supported by strong scientific evidence however we argue that the lack of evidence originates from shortcomings in the methodological approach to these paradigms. In our commentary, we argue for a redefinition of the paradigms rather than defining two 'new' paradigms. A better methodological approach and definitions of the paradigms based on the current evidence are needed rather than to abandon them.

\section{Keywords:}

IImpact - pronation - muscle activation - running - injury - performance

Citation:

Vanwanseele, B., Zhang X., Schütte, K. (2018): Muscle tuning and preferred movement path: do we need a paradigm shift or should we redefine the old? - comment on Nigg et al. Current Issues in Sport Science, 3:106. doi: 10.15203/CISS_2018.106

This is a commentary on a CISS report article authored by Nigg, B. M., Mohr, M. \& Nigg, S. R. (2017). Muscle tuning and preferred movement path - a paradigm shift. Current Issues in Sport Science, 2:007. doi: 10.15203/CISS_2017.007

\section{Introduction}

Over the last 40 years, Nigg and his colleagues have made tremendous contributions to the field of footwear biomechanics. They have invested a lot of research and energy in trying to confirm the two"old" paradigms. However, research, novel measurement and analysis techniques have led to new insights and the authors are to be recognized for their courage to step away from the traditional paradigm. We agree with Nigg that good biomechanical and epidemiological evidence behind the pronation and impact paradigm, as defined in the paper, is still scarce. It is however, our opinion that the current lack of evidence is partially due to methodological problems related to the definition and measurement of impact and pronation. We believe that we don't need to abandon the old paradigm but need to redefine them using the current scientific knowledge and new methodological approaches.

\section{Impact Forces}

Although large prospective studies investigating the link between vertical loading rate and running injuries are lacking, there is some evidence supporting this paradigm. For example, a recent gait retraining intervention study decreasing the vertical loading rates found a decrease in injury risk in novice runners by approximately $22 \%$ (Chan et al., 2018). In addition, a recent systematic review (Van Der Worp, Vrielink, \& Bredeweg, 2016) supports the association between loading rate and bone related injuries but not with all injuries together. 
Therefore, we believe that methodological problems hamper evidence for the impact paradigm especially the quantification of the loading and the definition of injuries. Using external loading as an estimate of internal tissue loading is limited and more objective ways to estimate internal loading are available. Musculoskeletal modelling and finite element modelling can provide data on internal loading of the musculoskeletal tissue. We acknowledge that these methods require specific expertise, are time consuming, making them less feasible for large cohort studies. However, research combining these models with easier to measure parameters should give further insight in quantifying internal loading. In addition, different tissue are also sensitive to different type of loading. Impact load and rate of loading might be relevant for bone tissue (Edwards, Ward, Meardon, \& Derrick, 2009), while muscle activation or moments are more relevant for muscle, ligament and tendinous tissues (Giddings, Beaupre, Whalen, \& Carter, 2000).

\section{Muscle Tuning paradigm}

In the new muscle tuning paradigm, it is suggested that if the frequency of the external loading is too close to the natural frequency of the soft tissue, muscle activation could reduce soft tissue vibration. Although this is an innovative and interesting concept, similar to the "old" paradigms, it currently lacks scientific evidence especially in running. Furthermore, no experimental or computational studies link tissue vibration with running injuries or performance. The mechanism that muscle activity might change the resonance of the input signal seems to be logical and theoretically sound, however how does vibration of the tissue lead to injury? In this paradigm, more muscle activity will reduce the vibration. Nevertheless, is this increased muscle activation protective against injuries? This somehow seems to be in contrast to the other new "Preferred Movement Path" paradigm, later discussed in this article.

\section{Pronation paradigm}

Similar to the loading paradigm, the pronation paradigm is hampered by methodological issues especially in the definition of foot pronation. Foot pronation is a multi-segment triplanar motion, consisting of: subtalar eversion, ankle dorsiflexion and forefoot abduction. However, the majority of early kinematic studies modelled the foot as one rigid segment, and therefore, primarily used rearfoot eversion as a measure of foot pronation (Hintermann \& Nigg, 1998). As a consequence, controlling excessive rearfoot eversion has become the focus of many interventions in people with pronated feet (Bishop, Arnold, \& May, 2016). We agree with Nigg that the scientific evidence for the relationship between rearfoot eversion and injury risk is weak. We, for instance, found that recreational runners with pronated feet remained asymptomatic despite a larger rearfoot eversion during gait compared to their counterparts with neutral feet
(Zhang, Aeles, \& Vanwanseele, 2017). This raises doubts on rearfoot eversion as indicator for overuse injury risk. However, using a multi-segment foot model, we demonstrated that symptomatic runners have larger transverse plane forefoot motion (unpublished data). As forefoot abduction is a component of foot pronation, it does not seem appropriate to abandon the pronation paradigm but rather to adopt a better definition and use multi-segment foot models to investigate this paradigm further. Moreover, interventions targeted at foot pronation should take into account the complex nature of the foot and not only focus on rearfoot eversion.

\section{Preferred Movement Path}

The preferred movement path is defined as a movement path that an individual chooses due to either minimal resistance, maximal comfort, or least energy demand (Nigg et al., 2017; Nigg, Baltich, Hoerzer, \& Enders, 2015). We believe that this paradigm is conceptually innovative in its break away from the traditional paradigms and its novel outlook on movement control. However, as it currently stands, we feel that this paradigm is still very much in its research infancy and requires more clarity. We would also argue that the word 'preferred' 1) qualitatively needs a better description and 2) quantitatively needs better criteria to set critical thresholds. As such, we analyze the preferred movement path by posing a few questions, and where possible, suggest clarification of the paradigm to direct future research.

\section{Qualitatively, what is "preferred?"}

On the one hand, preferred could refer to 'natural', that is, the unshod barefoot condition. On the other hand, 'preferred' could also refer to 'habitual', that is, the particular footwear or running surface condition that the individual has experience with and thus has become trained to or adapted to. Adoption of one of these opposing terms may improve the interpretation as to what constitutes the baseline of the 'preferred' condition. As a thought experiment, consider a runner who trains in multiple types of footwear and on multiple terrains. How would one define this particular runner's 'preferred' movement path? A 'habitually-preferred' movement path would essentially be one that is highly dynamic with an ever-changing baseline, a challenging feat to quantify and thus one that we would not advocate. Hence, to avoid ambiguity, we would avoid using 'habitually-preferred' and rather suggest a 'naturally-preferred' movement path.

Conceptually, the barefoot condition is innate to every individual. Methodologically, barefoot is unconstrained to any footwear characteristics, and therefore easy to standardize or generalize across individuals and laboratories. This 'naturallypreferred' condition could provide a good baseline to classify individual's into what Nigg coined as 'functional groups'. For instance, grouping individuals by evaluating their kinematic 
response to particular footwear types versus barefoot as the baseline. Therefore, the optimal footwear condition would be the one where the kinematic response deviates the least from natural condition.

\section{Quantitatively, what is "preferred?"}

Firstly, how much deviation from 'preferred' is too much? In a recent paper, Nigg (Nigg et al., 2017) arbitrarily set 2, 3 , and 5 degrees as thresholds for staying within the preferred movement path between running footwear conditions. However, it is not clear whether the magnitudes of these thresholds in absolute terms are generalizable between joints and planes of motion. For example, one could argue that 3.7 degrees change in ankle adduction is quite large compared to the same change in knee flexion. Interestingly, one study (Schrödter, Brüggemann, Hamill, \& Rohr, 2016) has provided a first step towards functional meaning of the paradigm by demonstrating that knee extensor strength was associated with the footwear-related variability (as a measure of deviations from the preferred movement path) of the hip and knee while running in six different types of footwear. However, what appears to be lacking are studies that can identify a 'critical' threshold in relation to injury risk, both retrospectively and prospectively.

Secondly, what specifically is the most appropriate measure to quantify 'deviation' from one's preferred movement path? Nigg (Nigg et al., 2017) used the mean absolute difference while other researchers have used the magnitude of "footwearrelated variability" for a given joint angle or moment during stance phase (Schrödter, Brüggemann, Hamill, \& Rohr, 2016). Recently, our research group has shown that other measures (e.g., acceleration waveform complexity, variability, stability) could alternatively be used to quantify deviations in a runner's movement path in relation to outdoor running fatigue and injury history (Schütte, Seerden, Venter, \& Vanwanseele, 2017). Future research is therefore encouraged to explore other measures that could shed additional light into determining a runner's movement 'path' in the real world.

\section{Funding}

The authors have no funding or support to report.

\section{Competing Interests}

The authors have declared that no competing interests exist.

\section{Data Availability Statement}

All relevant data are within the paper.

\section{References}

Bishop, C., Arnold, J. B., \& May, T. (2016). Effects of Taping and Orthoses on Foot Biomechanics in Adults with Flat-Arched Feet. Medicine \& Science in Sports \& Exercise, 48(4), 689696. https://doi.org/10.1249/MSS.0000000000000807

Chan, Z. Y. S., Zhang, J. H., Au, I. P. H., An, W. W., Shum, G. L. K., Ng, G. Y. F., \& Cheung, R. T. H. (2018). Gait Retraining for the Reduction of Injury Occurrence in Novice Distance Runners 1-Year Follow-up of a Randomized Controlled Trial. The American Journal of Sports Medicine, 46(2), 388-395. https://doi.org/10.1177/0363546517736277

Edwards, W. B., Ward, E. D., Meardon, S. A., \& Derrick, T. R. (2009). The Use of External Transducers for Estimating Bone Strain at the Distal Tibia During Impact. Journal of Biomechanical Engineering, 131, 1-6. https://doi. org/10.1115/1.3118762

Giddings, V. L., Beaupre, G. S., Whalen, R. T., \& Carter, D. R. (2000). Calcaneal loading during walking and running. Medicine \& Science in Sports \& Exercise, 32(3), 627-634.

Hintermann, B., \& Nigg, B. M. (1998). Pronation in runners: Implications for injuries. Sports Medicine, 26(3), 169-176. https://doi.org/10.2165/00007256-199826030-00003

Nigg, B. M., Baltich, J., Hoerzer, S., \& Enders, H. (2015). Running shoes and running injuries: mythbusting and a proposal for two new paradigms: "preferred movement path " and " comfort filter ." British Journal of Sports Medicine, 49, 1290-1294. https://doi.org/10.1136/bjsports-2015-095054

Nigg, B. M., Vienneau, J., Smith, A. C., Trudeau, M. B., Mohr, M., \& Nigg, S. R. (2017). The Preferred Movement Path Paradigm: Influence of Running Shoes on Joint Movement '. Medicine \& Science in Sports \& Exercise, (15), 1641-1648. https://doi.org/10.1249/MSS.0000000000001260

Schrödter, E., Brüggemann, G., Hamill, J., \& Rohr, E. (2016). Footwear-related variability in running. Footwear Science, 8(1), 23-32. https://doi.org/10.1080/19424280.2016.1142002

Schütte, K. H., Seerden, S., Venter, R., \& Vanwanseele, B. (2017). Influence of outdoor running fatigue and medial tibial stress syndrome on accelerometer-based loading and stability. Gait \& Posture, 59(March 2017), 222-228. https:// doi.org/10.1016/j.gaitpost.2017.10.021

Van Der Worp, H., Vrielink, J. W., \& Bredeweg, S. W. (2016). Do runners who suffer injuries have higher vertical ground reaction forces than those who remain injury-free? A systematic review and meta-analysis. British Journal of Sports Medicine, 50(8), 450-457. https://doi.org/10.1136/ bjsports-2015-094924

Zhang, X., Aeles, J., \& Vanwanseele, B. (2017). Comparison of foot muscle morphology and foot kinematics between recreational runners with normal feet and with asymptomatic over-pronated feet. Gait and Posture, 54. https:// doi.org/10.1016/j.gaitpost.2017.03.030 